\title{
Bose-Einstein condensation of pions in heavy-ion collisions at the CERN Large Hadron Collider (LHC) energies
}

\author{
Viktor Begun ${ }^{1,2}$ and Wojciech Florkowski ${ }^{3}$ \\ ${ }^{1}$ Institute of Physics, Jan Kochanowski University, PL-25406 Kielce, Poland \\ ${ }^{2}$ Bogolyubov Institute for Theoretical Physics, 03680 Kiev, Ukraine \\ ${ }^{3}$ The H. Niewodniczański Institute of Nuclear Physics, \\ Polish Academy of Sciences, PL-31342 Kraków, Poland
}

(Dated: July 6, 2018)

\begin{abstract}
We analyse in detail the possibility of Bose-Einstein condensation of pions produced in heavy-ion collisions at the beam energy $\sqrt{s_{\mathrm{NN}}}=2.76 \mathrm{TeV}$. Our approach is based on the chemical non-equilibrium thermal model of hadron production which has been generalised to include separately the contribution from the local zero-momentum state. In order to study both the hadronic multiplicities and the transverse-momentum spectra, we use the Cracow freeze-out model which parameterises the flow and space-time geometry of the system at freeze-out in a very economic way. Our analysis indicates that about $5 \%$ of all pions may form the Bose-Einstein condensate.
\end{abstract}

PACS numbers: 25.75.-q, 25.75.Dw, 25.75.Ld

Keywords: relativistic heavy-ion collisions, thermal models of hadron production, Bose-Einstein condensation, Large Hadron Collider (LHC) 


\section{INTRODUCTION}

Thermal models of hadron production serve us as the basic tool to obtain general information about the properties of matter produced in heavy-ion collisions [1 25]. The successes of such models in description of different collision processes indicate typically that the produced matter is well thermalised and exhibits collective behavior. This, in turn, suggests the formation of the equilibrated system of quarks and gluons at earlier stages of the collisions.

The newest LHC data on hadron production indicates that the most common thermal approach, based on the chemical and thermal equilibration assumption, fails to describe the ratio of protons to pions [26]. The mean multiplicities of protons and anti-protons also deviate from the fit for about three standard deviations [27]. In addition, most of the hydrodynamic calculations, that have been successfully used to reproduce the harmonic flow coefficients, have problems with the correct predictions for very low transverse-momentum spectra of pions [26, 28, 30].

In our previous works [31, 32] we have demonstrated that these two problems may be solved by the assumption that matter produced in heavy-ion collisions at the LHC energies is formed out of chemical equilibrium [1, 3, 14]. One of the predictions of the chemical non-equilibrium model is that the pion abundances are characterized by the non-zero value of the chemical potential $^{1}$ which is very close to the critical value for the Bose-Einstein condensation (BEC). This suggests a possible onset of BEC at the LHC energies.

The recent analysis of two- and three-pion correlations done by ALICE collaboration [34] shows an intriguing result that a coherent fraction in charged pion emission may reach $23 \%$. The main aim of our present work is to determine quantitatively the amount of particles in condensate at different centralities using mean multiplicities and spectra of the particles.

The fact that the pion chemical potential is very close to its critical value requires that thermal analyses in this case should be performed in a more careful way with the explicit treatment of the local zero-momentum state [35]. As the inclusion of the pion ground state effects is relatively easy in the analyses of the ratios of hadronic abundances, a fully covariant framework describing local formation of the condensate in an expanding medium is not at hand. The formulation of such a framework requires that the Cooper-Frye formula is generalized in a very special way. In this work we demonstrate how this can be achieved for boost-invariant and cylindrically symmetric systems. We make the corresponding changes in the previously

\footnotetext{
${ }^{1}$ The equilibrium model [33] also shows the hints of pion chemical potential at the LHC.
} 
used by us SHARE [36] and THERMINATOR [37, 38] codes.

The hadronic data are analyzed within three possible scenarios: using the full equilibrium model (EQ), the chemical non-equilibrium model (NEQ) that have been used in [31, 32], and the newly developed chemical non-equilibrium model with Bose-Einstein condensate (BEC) ${ }^{2}$. We include all available LHC data on $\mathrm{Pb}+\mathrm{Pb}$ collisions at $\sqrt{s_{\mathrm{NN}}}=2.76 \mathrm{TeV}$ [26, 39 41] in our study using the modified SHARE and use the modified THERMINATOR in order to check the effect of BEC on the pion spectra at low transverse-momenta [26, 42]. We also discuss in greater detail the effects which the data on neutral pion production may have on our results. Our comparisons between different thermal frameworks indicate the plausibility of pion condensation in heavy-ion collisions at the LHC.

The paper is organized as follows: in Section II we describe the role of the ground state and the difference between EQ, NEQ and BEC models. In Section III we explain the method of our analysis. Sections [V] and $\mathrm{V}$ show the results of the analysis of mean multiplicities and particle spectra, correspondingly. Section VI concludes the paper.

\section{EXPLICIT TREATMENT OF HADRONIC GROUND STATES}

\section{A. Ground state contribution}

Calculating particle multiplicities in a non-interacting quantum gas, one changes the summation over discrete momentum levels by the integration over the continuous spectrum

$$
\begin{aligned}
N & =\sum_{n} \frac{g_{n}}{\exp \left(\frac{\sqrt{p_{n}^{2}+m^{2}}-\mu}{T}\right) \mp 1} \simeq \int \frac{d^{3} x d^{3} p}{h^{3}} \frac{g}{\exp \left(\frac{\sqrt{\mathbf{p}^{2}+m^{2}}-\mu}{T}\right) \mp 1} \\
& =V \int_{0}^{\infty} \frac{d^{3} p}{(2 \pi)^{3}} \frac{g}{\exp \left(\frac{\sqrt{\mathbf{p}^{2}+m^{2}}-\mu}{T}\right) \mp 1} .
\end{aligned}
$$

Here $g_{n}$ is the degeneracy of the $p_{n}$-th momentum state, $T$ is the system temperature, $m$ is the mass of particles, and $\mu$ is the chemical potential. The integral over space coordinates gives the volume of the system $V$, and the factor $(2 \pi)^{3}$ appears since we use the natural units where $h \equiv 2 \pi \hbar$ and $\hbar=1$. For simplicity, we do not specify the physical character of the chemical potential $\mu$ at the moment and consider only one type of particles.

\footnotetext{
${ }^{2}$ Note that we use the same acronym to denote both the Bose-Einstein condensation and our framework which explicitly includes the condensate formation.
} 
If the chemical potential approaches the value of the particle mass, the integrand in the second line of Eq. (1) stays constant for $\mathbf{p} \rightarrow 0$, because the singularity of the denominator is cancelled by the integration measure $d^{3} p$. However, the first term in the sum over quantum levels becomes infinite in the limit $\mu \rightarrow m$ at $\mathbf{p}=0$

$$
N_{\text {cond }}=\frac{g_{0}}{\exp \left(\frac{m-\mu}{T}\right)-1} \rightarrow \infty \quad \text { for } \quad \mu \rightarrow m .
$$

Therefore, at the onset of BEC, when $\mu \rightarrow m$, one should keep the summation over the low momentum states and start the integration in (1) at $|\mathbf{p}|>0$. One can show, however, that in the thermodynamic limit, $V \rightarrow \infty$, one may separate the $\mathbf{p}=0$ term only and start the integration from zero [43], namely

$$
\begin{aligned}
N & \simeq \frac{g}{\exp \left(\frac{m-\mu}{T}\right)-1}+V \int_{0}^{\infty} \frac{d^{3} p}{(2 \pi)^{3}} \frac{g}{\exp \left(\frac{\sqrt{\mathbf{p}^{2}+m^{2}}-\mu}{T}\right)-1} \\
& =N_{\text {cond }}+N_{\text {norm }}=N_{\text {cond }}+V n_{\text {norm }} .
\end{aligned}
$$

The degeneracy of the zero level is the same as that of the continuous spectrum, $g_{0}=g=2 s+1$, where $s$ is the particle spin.

\section{B. Chemical potentials in a hadron gas}

In the case of a hadron-resonance gas the above simple picture becomes more complicated one should include: the sum over all existing hadron states, the integral over the mass spectrum of broad resonances, and the decays of resonances defined by their branching ratios. In addition, the chemical potential is different for each particle type $i$. In the case of the EQ model, the chemical potential becomes a linear combination of the particle's electric, $Q_{i}$, baryon, $B_{i}$, and strange, $S_{i}$, charges

$$
\mu_{i}^{\mathrm{EQ}}=Q_{i} \mu_{Q}+B_{i} \mu_{B}+S_{i} \mu_{S} .
$$

The chemical potentials $\mu_{Q}, \mu_{B}$ and $\mu_{S}$ are the same in the whole system. They control the conservation of electric charge, baryon number, and strangeness. In the midrapidity region at the LHC these quantities are negligible, so it is reasonable to assume that

$$
\mu_{i}^{\mathrm{EQ}} \simeq 0
$$

The charge conservation is connected with the conservation of the difference of the quark and antiquark numbers. However, a fast expansion and cooling of the quark-gluon plasma may 
lead to the effective conservation of the sum of quarks and antiquarks [3]. It can happen if the hadronization is so fast, that the quarks do not have enough time to annihilate and lower their abundances to the equilibrium values at the chemical freeze-out temperature. This leads to the appearance of additional non-equilibrium chemical potential

$$
\mu_{i}^{\mathrm{NEQ}}=\left(N_{q}^{i}+N_{\bar{q}}^{i}\right) \mu_{q}+\left(N_{s}^{i}+N_{\bar{s}}^{i}\right) \mu_{s}
$$

where $N_{q}^{i}$ and $N_{\bar{q}}^{i}$ are numbers of light $(u, d)$ quarks and antiquarks, while $N_{s}^{i}$ and $N_{\bar{s}}^{i}$ are the numbers of strange quarks and antiquarks in the $i$ th hadron. For example, the non-equilibrium chemical potentials for pions, protons, kaons, and $\Lambda$ 's read:

$$
\mu_{\pi}=2 \mu_{q}, \quad \mu_{p}=3 \mu_{q}, \quad \mu_{K}=\mu_{q}+\mu_{s}, \quad \mu_{\Lambda}=\mu_{q}+2 \mu_{s} .
$$

We neglect the contributions from heavier quarks, because they are suppressed by the Boltzmann factor.

The non-equilibrium chemical potentials may be encoded in the fugacity factors of the form

$$
\Upsilon_{i} \equiv \exp \left(\frac{\mu_{i}^{\mathrm{NEQ}}}{T}\right)=\gamma_{q}^{N_{q}^{i}+N_{\bar{q}}^{i}} \gamma_{s}^{N_{s}^{i}+N_{\bar{s}}^{i}}
$$

As a matter of fact, the non-equilibrium hypothesis of hadron production at freeze-out was originally proposed in terms of $\Upsilon_{i}$ 's [1], therefore, we will follow the tradition and use $\gamma_{q}$ and $\gamma_{s}$ instead of $\mu_{q}$ and $\mu_{s}$. We have introduced the condensation term $N_{\text {cond }}$ given by Eq. (3) in the latest version of SHARE [36]. The calculations done in this way are denoted below as the BEC case.

\section{FITTING STRATEGY}

\section{A. Centrality selection}

There are several technical issues that we have to address now. Different particles are measured in different centrality bins. This leads to problems with the interpretation of the data. The authors of Ref. [44] solved this problem by redistributing heavy strange particles in smaller centrality bins, in which light particles such as pions are measured. However, we have

found that this strategy introduces an uncertainty in the thermal analyses, which leads to large errors for the output parameters, especially for $\gamma_{q}$ and $\gamma_{s}$.

Therefore, herein we do the opposite and merge the particles measured with the finer centrality steps into larger centrality sets at which strange particles are measured. This gives us 
six centrality intervals: $0-10 \%, 10-20 \%, 20-40 \%, 40-60 \%, 60-80 \%$, and $80-90 \%$. The corresponding errors are recalculated following the standard procedure of error propagation.

\section{B. Multiplicities vs. ratios}

The experiment provides both multiplicities (strictly speaking the rapidity densities) and particle ratios. Some authors prefer to fit particle ratios instead of multiplicities, in order to have fewer number of parameters and to get rid of the system's volume. However, one should be careful with this procedure, especially for the non-equilibrium models, because with given $n$ multiplicities one may construct $n(n-1) / 2$ ratios. This gives quite substantial number of ratios for large $n$, which are correlated.

In practice, one usually restricts oneself to a smaller and experimentally available set of ratios, and thus studies only a sub-domain of the whole system. This strategy affects the results, in particular for the NEQ models, where each particle has its own pre-factor $\Upsilon_{i}(8)$. For example, the multiplicity of protons is approximately proportional to $\Upsilon_{p}=\gamma_{q}^{3}$, while that of pions to $\Upsilon_{\pi}=\gamma_{q}^{2}$. Therefore the ratio of protons to pions will give us the factor $\gamma_{q}$, which is much less sensitive to the change of $\gamma_{q}$ than the mean multiplicities of protons and pions. The ratio of lambda to kaon cancels the information on strangeness, $\Upsilon_{\Lambda} / \Upsilon_{K}=\left(\gamma_{q}^{2} \gamma_{s}\right) /\left(\gamma_{q} \gamma_{s}\right)=\gamma_{q}$, and gives us as much information as proton to pion ratio, etc. Therefore, even if one takes all $n(n-1) / 2$ ratios, one may get different temperatures and other parameters for the same set of $n$ particles.

Another important aspect of the analysis is that ALICE measures only the rapidity densities. Below, we argue that Eq. (3) can be transformed in this case to the form

$$
\frac{\Delta N}{\Delta y}=\frac{d V}{d y} n_{\text {norm }}+N_{\text {cond }},
$$

where $d V / d y$ is the volume of the produced particles per unit rapidity. Note that the volume does not cancel in the ratios of the rapidity densities in the case with the condensate included.

\section{Neutral pion effects on the fits}

The BEC model gives typically a very good fit for all observed particles. However, the calculated number of the not yet measured $\pi^{0}$ mesons is extremely large. This is so, because the fit suggests a high value of $\gamma_{q}$, at which the neutral pions, as the lightest particles, condense 
first. This behaviour suggests that one should make a reasonable estimate of the $\pi^{0}$ multiplicity and use it as an input in the BEC fit.

The isospin symmetry of strong interactions suggests that the number of $\pi^{0}$ 's must be the same as the number of positively or negatively charged pions. Although the isospin symmetry is in fact slightly broken (in particular, $\pi^{0}$ is lighter than $\pi^{ \pm}$) we neglect this effect in order to get a lower bound for the amount of condensate, and use the constraint

$$
N_{\pi^{0}}(c)=\frac{1}{2}\left(N_{\pi^{+}}(c)+N_{\pi^{-}}(c)\right)
$$

Equation 10 represents an estimate of a possible production rate. The error bars are recalculated as follows

$$
\Delta N_{\pi^{0}}(c)=\frac{1}{2}\left(\Delta N_{\pi^{+}}(c)+\Delta N_{\pi^{-}}(c)\right)
$$

Since the experimental errors for the $\pi^{+}$and $\pi^{-}$mean multiplicities are practically the same, we obtain almost the same error bars for $\pi^{0}$. We note that it is much more difficult to measure $\pi^{0}$ 's than charged pions. At present, the spectrum for $p_{T}>700 \mathrm{MeV}$ is known only. It gives just about $1 / 3$ of all expected $\pi^{0}$ s. In the $p_{T}$ range where both $\pi^{ \pm}$and $\pi^{0}$ are measured, the latter has about twice bigger relative errors. The error bars for $\pi^{0}$-s also increase fast, while going from high to low $p_{T}$ in the existing data [42]. Therefore, we think that (11) is a reasonable estimate.

\section{FIT OF HADRONIC MULTIPLICITIES}

We perform our analysis using the rapidity densities of $\pi^{ \pm}, p, \bar{p}, K^{ \pm}, K_{S}^{0}, \Lambda, \phi, \Xi^{ \pm}$and $\Omega^{ \pm}$. In order to include the effect of the neutral pions on the fits, we also include the estimate based on Eqs. (10) and (11). This gives us 14 data points for the centralities 0-10\%, 10-20\%, 20-40\%, 40-60\%, 60-80\%, and 10 data points for the centrality bin 80-90\%, where $\Xi^{ \pm}$and $\Omega^{ \pm}$ are not measured. We do not include the short-living $K^{*}(892)^{0}$ [39] - in this way we may check if it follows the pattern obtained for other long-living particles. By doing so we further examine the applicability of the single freeze-out concept, see our discussion in [32].

Our results are shown in Figs. 1, 2 and 3. The volume determined from the fits is practically the same for the NEQ and BEC models, because the number of particles in the ground state appears to be relatively small. The EQ volume is larger, because $\Upsilon>1$ makes the system denser in NEQ and BEC, compared to EQ. Interestingly, the explicit treatment of the ground 

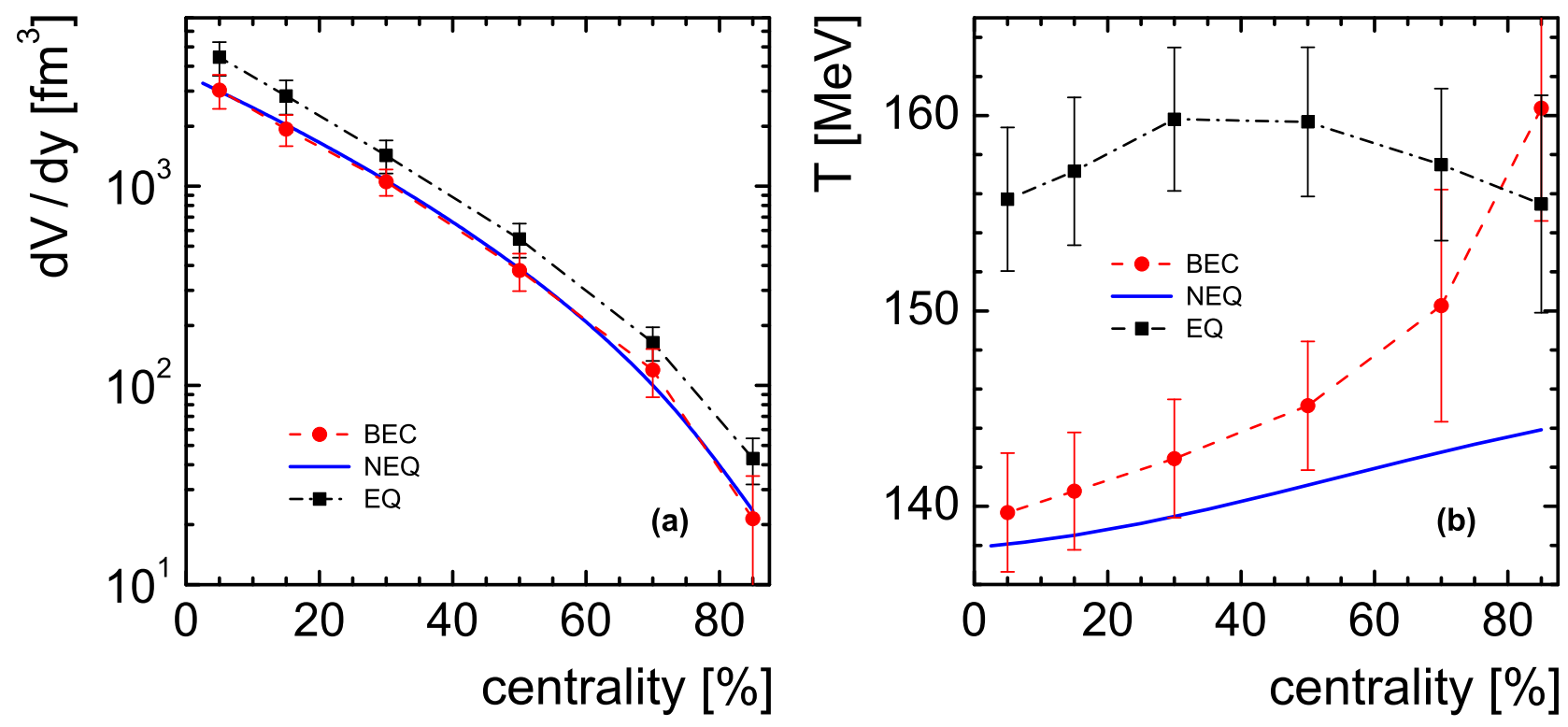

FIG. 1: (Color online) The left figure shows the system volume, while the right figure shows the system temperature obtained as the fit to mean multiplicities of hadrons, which are produced in $\mathrm{PbPb}$ collisions at the beam energy $\sqrt{s_{\mathrm{NN}}}=2.76 \mathrm{TeV}$. The BEC lines correspond to the non-equilibrium model with the Bose condensate in the ground state (3). The NEQ lines correspond to the nonequilibrium model without the Bose condensate, and the EQ lines are obtained for the equilibrium model. The NEQ lines are from the paper [32], while the EQ and BEC lines are from [35].

state lowers the gap in temperature between equilibrium and non-equilibrium models. These two versions of the model even coincide for the peripheral collisions, as it was shown before in 35 .

The left panel of Fig. 3 shows that the $\chi^{2}$ fit is much better for BEC than for EQ. Even the larger numbers of degrees of freedom in EQ $\left(N_{\text {dof }}=12\right.$ for $c<80 \%$ and $N_{\text {dof }}=8$ for $c=80-90 \%$ in EQ, versus 10 and 6 in BEC, respectively) do not help to decrease the $\chi^{2} / N_{\text {dof }}$ values. The agreement with the data for individual particles is also significantly better for BEC than for EQ. For example, the $K^{*}(892)^{0}$ resonance, which was not included in the fit, deviates from the data by more than $2 \sigma$ in most central collisions in EQ, similarly as in Ref. [27], while it agrees with the data within $1 \sigma$ in BEC for each centrality. The successful fit of the short living $K^{*}(892)^{0}$ with the parameters obtained for long living particles is a strong argument in favor of the single-freeze-out approximation.

The values of $\chi^{2} / N_{\text {dof }}$ even drop below unity in BEC, which means that the experimental errors are probably too large. To check this behaviour, we calculated the $\chi^{2} / N_{\text {dof }}$ contours and 

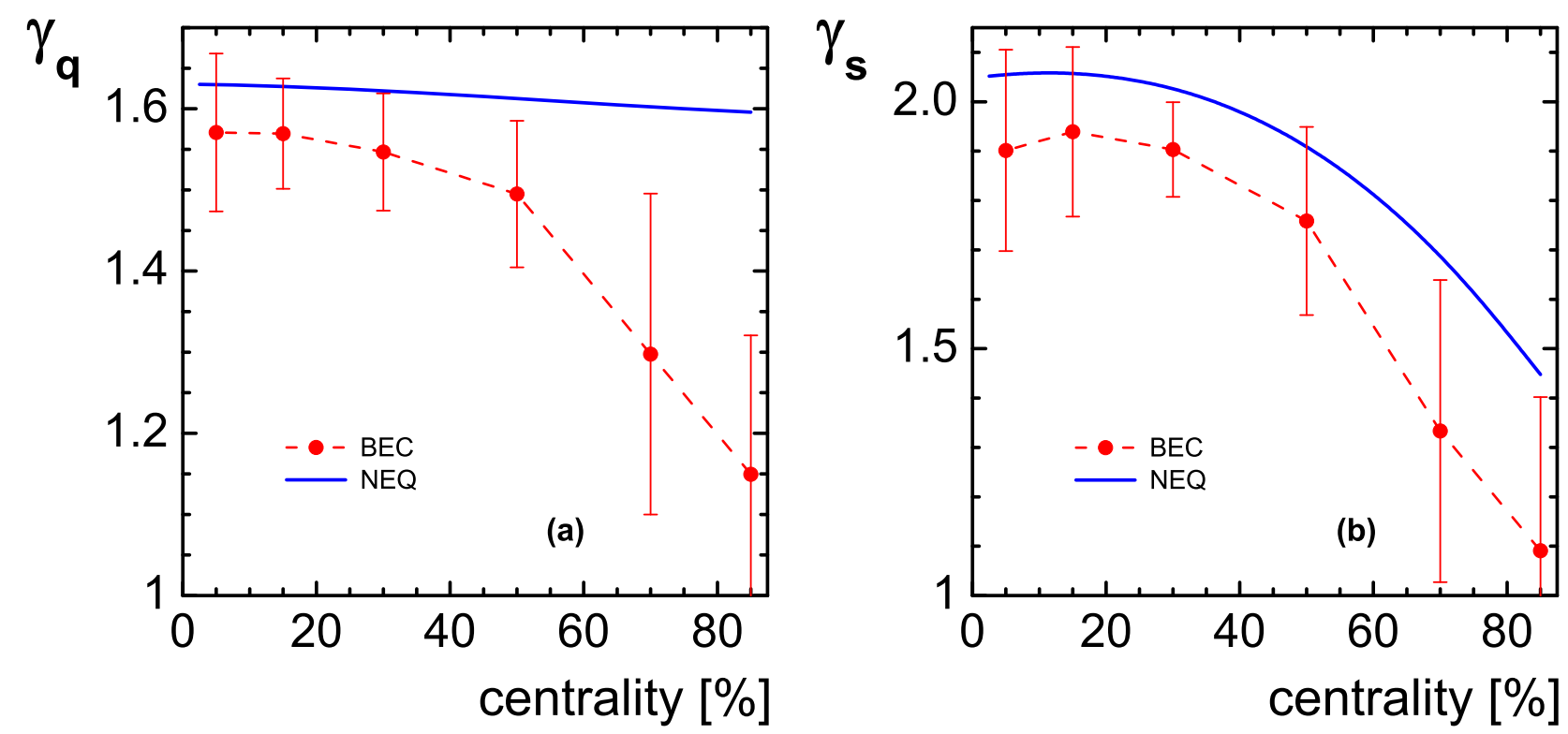

FIG. 2: (Color online) The non-equilibrium parameters $\gamma_{q}$ on the left and $\gamma_{s}$ on the right in BEC [35] and NEQ [32]. The corresponding EQ values are equal to unity by definition.

found that the minimum of $\chi^{2} / N_{\text {dof }}$ is very shallow in BEC. The right panel of Fig. 3 shows the $10 \%$ deviation from the points with the best fit in the $T-\gamma_{q}$ plane for BEC. The centrality
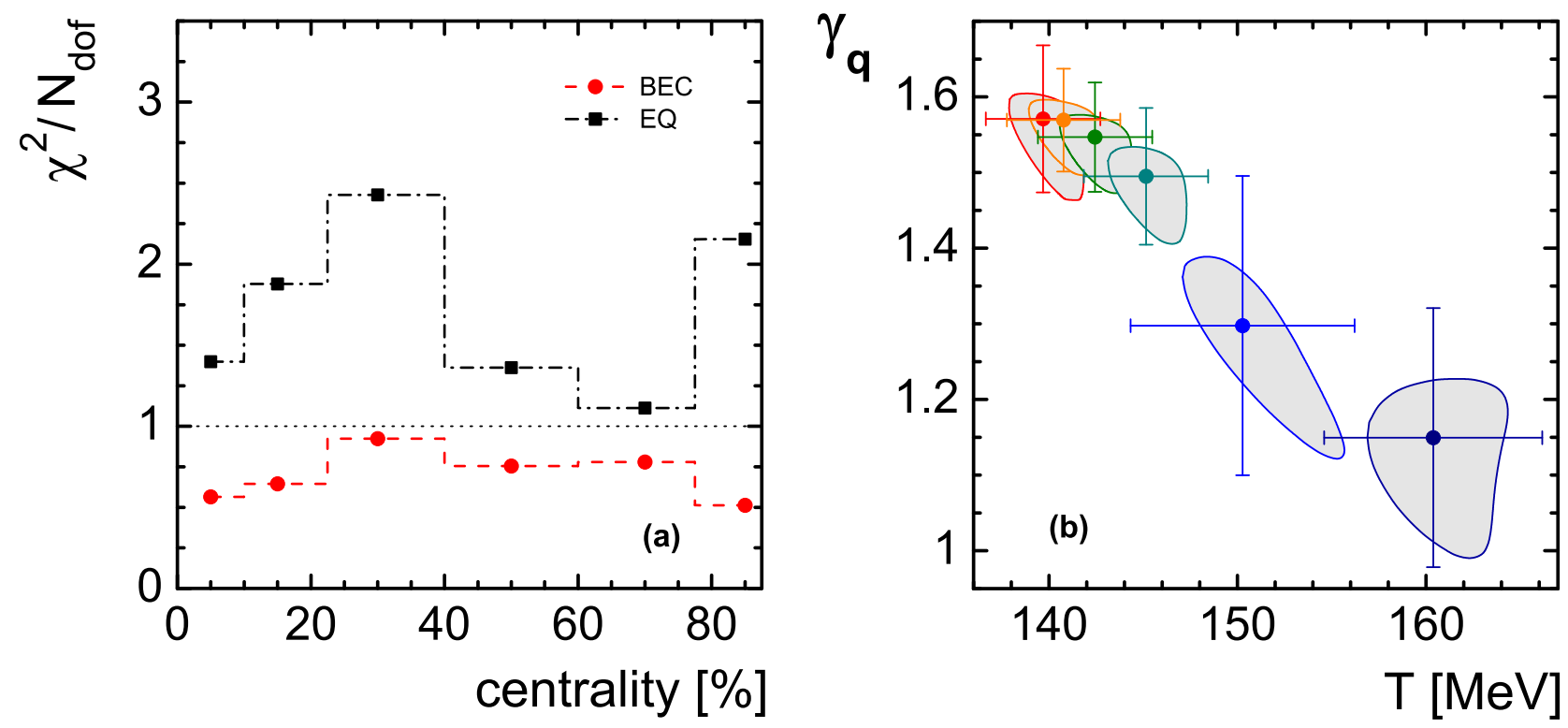

FIG. 3: (Color online) The $\chi^{2} / N_{\text {dof }}$ in BEC and EQ on the left, and the best fit values for $\gamma_{q}$ and $T$ in $\mathrm{BEC}$ on the right together with the error bars. The contours on the right figure indicate the area where $\chi^{2} / N_{\text {dof }}$ increases up to $10 \%$.

increases from lower to higher temperatures, as in Fig. 1. One may see that the increase of 
$\chi^{2} / N_{\text {dof }}$ by $10 \%$ determines the area that covers a substantial part of the region marked by the error bars. This is so because there is a sudden rise in the values of $\chi^{2} / N_{\text {dof }}$ for increasing $\gamma_{q}$. This effect elongates the contours towards smaller values of $\gamma_{q}$. One may also notice an anti-correlation between $\gamma_{q}$ and $T$.

The number of particles in the condensate in EQ, NEQ and BEC models can be calculated using Eq. (2) and our results obtained with this formula are presented in Fig. 4. The grey bands give the upper and lower bounds on the amount of condensate in BEC. The width of the bands is determined by the values of $T$ and $\gamma_{q}$ taken along the contours shown in Fig. 3 (other parameters are taken as the optimal).
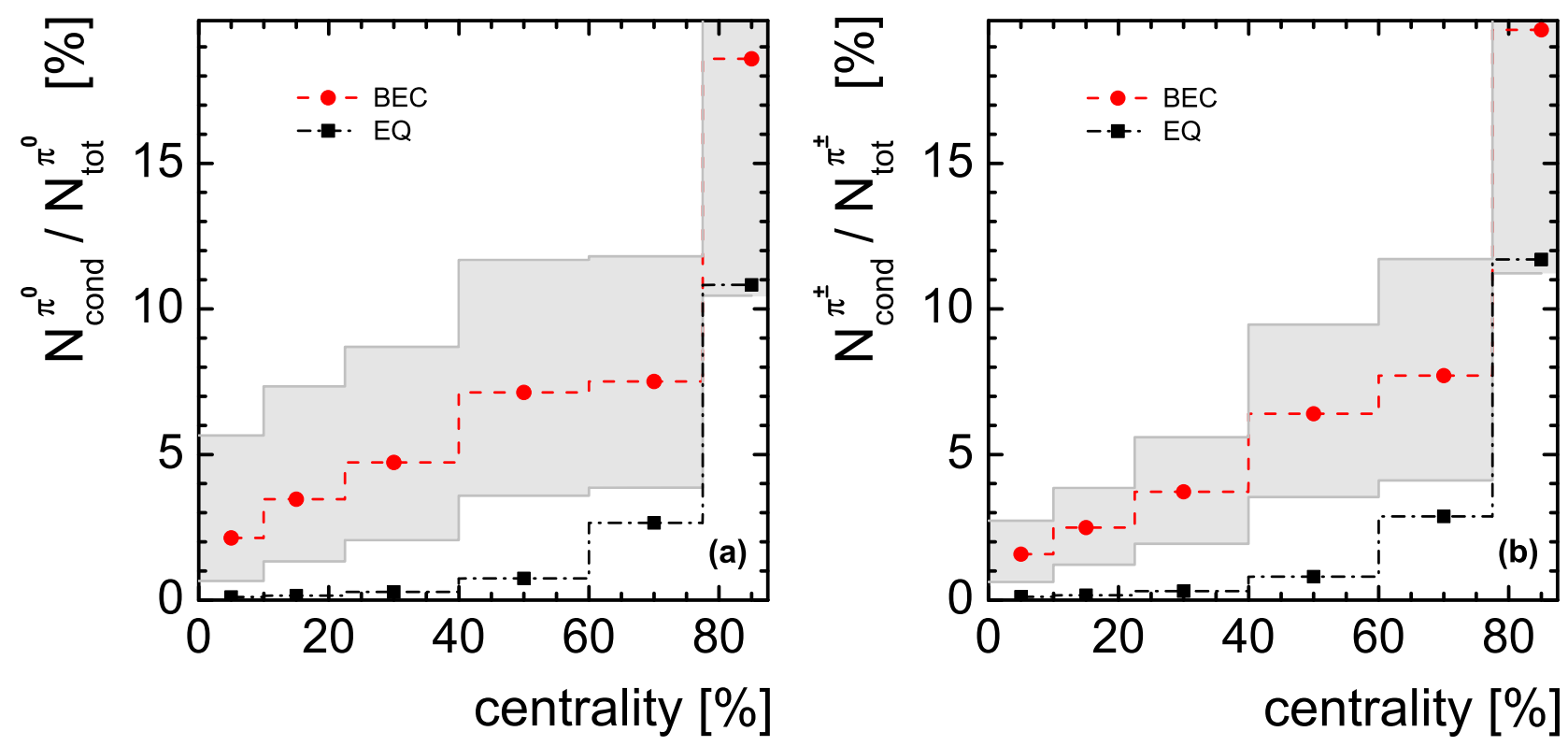

FIG. 4: (Color online) The ratio of neutral pions in the condensate on the ground state $N_{\text {cond }}^{\pi^{0}}$ to the total number of neutral pions $N_{\text {tot }}^{\pi^{0}}=N_{\text {cond }}^{\pi^{0}}+N_{\text {norm }}^{\pi^{0}}$ is shown on the left figure. The same for charged pions is shown on the right figure. The BEC lines were obtained using Eq. (3), while the EQ lines show the neglected contribution of the ground state in the EQ model, see Eq. (12).

The condensate rate in the NEQ model is not shown, as it is very large. It means that using NEQ model, one should always take into account the ground state effect. Otherwise, the obtained $\gamma_{q}$ and $\gamma_{s}$ would be too high, while temperature too small.

In BEC the condensate rate is $2 \%$ in the most central collisions and $7-8 \%$ in peripheral collisions (with a sudden increase to $19 \%$ in the most peripheral collisions). The plots for $\pi^{0}$ and $\pi^{ \pm}$are similar, because of the isospin symmetry that we demand with the use of Eqs. 100 and (11). The upper bound is higher for $\pi^{0}$ in the most central collisions, because temperature 
is lower there and the difference in masses of $\pi^{0}$ and $\pi^{ \pm}$becomes more important.

The increase of the pion condensate contribution with centrality in the BEC model is unexpected, because $\gamma_{q}$ and therefore $\mu_{\pi}$ decrease with centrality. However, it has been observed already before [43] that for smaller volumes the ratio of particles in the condensate increases if the system's temperature is sufficiently high. Therefore, the decrease of volume may produce the increase in the condensate rate.

We also note that the ratio of the $\pi^{0}$ and $\pi^{ \pm}$number in the zero momentum level (neglected in EQ calculations),

$$
N_{\text {cond }}^{\mathrm{EQ}}=\frac{1}{\exp \left(m_{\pi} / T_{\mathrm{EQ}}\right)-1},
$$

to the total number of $\pi^{0}$ and $\pi^{ \pm}$-s also increases for more than $10 \%$. The number of particles

in the ground state $N_{\text {cond }}^{\mathrm{EQ}} \simeq 0.7$ in 12 for all centralities, while $N_{\text {tot }}^{\mathrm{EQ}}$ decreases from about 700 to 7 at the most peripheral collisions, see [26] for the $\pi^{ \pm}$data. Therefore the ratio rises from $0.1 \%$ to $10 \%$. It means that the ground state should be included for the analysis of very peripheral heavy-ion or proton-proton collisions even in equilibrium models.

\section{SPECTRA}

\section{A. Covariant Cooper-Frye formula with the condensate}

Equation (3) can be formally obtained from (1) by inserting the distribution function which contains a delta function component

$$
f(\mathbf{p})=\frac{g}{\exp \left(\frac{\sqrt{\mathbf{p}^{2}+m^{2}}-\mu}{T}\right)-1}\left[1+\frac{(2 \pi)^{3}}{V} \delta^{(3)}(\mathbf{p})\right]
$$

in the integral over momentum (1).

In what follows we generalize Eq. 13 to the form suitable for hydrodynamic and kinetictheory applications. At first we note that the number of particles $N$ contained in the static box of volume $V$ may be obtained as the integral over the phase-space distribution function $\bar{f}(x, p)=\bar{f}(t, \mathbf{x}, \mathbf{p})$, namely

$$
N=\int d^{3} x d^{3} p \bar{f}(x, p)
$$

Hence, for the particles in a static box we may use the identification $\bar{f}(x, p)=(2 \pi)^{-3} f(\mathbf{p})$. If the particles are produced on the freeze-out hypersurface $\Sigma_{\mu}$, Eq. (14) is generalised to the 
form

$$
N=\int d \Sigma_{\mu} \int \frac{d^{3} p}{E_{p}} p^{\mu} \bar{f}(x, p) .
$$

Then, the covariant generalisation of $(13)$ for the systems which are boost-invariant and cylindrically symmetric is

$$
\bar{f}(x, p)=\frac{1}{(2 \pi)^{3}} \frac{g}{\exp \left(\frac{p \cdot U-\mu}{T}\right)-1}\left[1+\frac{(2 \pi)^{3}}{\mathcal{V}} \delta(p \cdot X) \delta(p \cdot Y) \delta(p \cdot Z)\right] .
$$

Here $\mathcal{V}=\int d \Sigma_{\mu} u^{\mu}$ is the Lorentz invariant volume of the system, where the condensate is created. The quantity $U$ is the four-velocity of the fluid element at freeze-out, while the fourvectors $X, Y$, and $Z$ define the three spatial directions in the fluid local rest frame. In the center-of-mass system of the colliding nuclei, the form of the four-vectors $X, Y$, and $Z$ is [45]:

$$
\begin{aligned}
X & =\left(\sinh \theta_{T} \cosh \eta_{\|}, \cosh \theta_{T} \cos \phi, \cosh \theta_{T} \sin \phi, \sinh \theta_{T} \sinh \eta_{\|}\right) \\
Y & =(0,-\sin \phi, \cos \phi, 0) \\
Z & =\left(\sinh \eta_{\|}, 0,0, \cosh \eta_{\|}\right) .
\end{aligned}
$$

Here $\theta_{T}$ is the transverse rapidity of the fluid element, $\phi$ is the azimuthal angle, and $\eta_{\|}$is the space-time rapidity. In the local rest frame of the fluid element we have: $X=(0,1,0,0)$, $Y=(0,0,1,0), Z=(0,0,0,1)$. The projections of the four-momentum along $X, Y$, and $Z$ are

$$
\begin{aligned}
& p_{X}=p \cdot X=m_{T} \sinh \theta_{T} \cosh \left(y-\eta_{\|}\right)-p_{T} \cosh \theta_{T} \cos \left(\phi_{p}-\phi\right), \\
& p_{Y}=p \cdot Y=p_{T} \sin \left(\phi-\phi_{p}\right) \\
& p_{Z}=p \cdot Z=m_{T} \sinh \left(\eta_{\|}-y\right)
\end{aligned}
$$

Here we used the parameterisation of the four-momentum: $p^{\mu}=\left(p^{0}, p^{1}, p^{2}, p^{3}\right)=$ $\left(m_{T} \cosh y, p_{T} \cos \phi_{p}, p_{T} \sin \phi_{p}, m_{T} \sinh y\right)$ where $p^{0}=E_{p}=\sqrt{\mathbf{p}^{2}+m^{2}}$ is the energy of a particle, $y$ and $\phi_{p}$ are the particle rapidity and azimuthal angle of the momentum, $p_{T}=$ $\sqrt{\left(p^{1}\right)^{2}+\left(p^{2}\right)^{2}}$ is the transverse momentum, and $m_{T}=\sqrt{p_{T}^{2}+m^{2}}$ - the transverse mass.

The Lorentz covariant integral in 150 is

$$
N^{\mu}=\int \frac{d^{3} p}{E_{p}} p^{\mu} \frac{1}{(2 \pi)^{3}} \frac{g}{\exp \left(\frac{p \cdot U-\mu}{T}\right)-1}\left[1+\frac{(2 \pi)^{3}}{\mathcal{V}} \delta(p \cdot X) \delta(p \cdot Y) \delta(p \cdot Z)\right] .
$$

Because of the Lorentz covariance and quadratic dependence of the Bose-Einstein distribution of three-momentum, one can conclude that $N^{\mu}=n U^{\mu}$ with the density $n$ given by the expression

$$
n=\int d^{3} p \frac{1}{(2 \pi)^{3}} \frac{g}{\exp \left(\frac{\sqrt{\mathbf{p}^{2}+m^{2}}-\mu}{T}\right)-1}\left[1+\frac{(2 \pi)^{3}}{\mathcal{V}} \delta\left(p_{x}\right) \delta\left(p_{y}\right) \delta\left(p_{z}\right)\right] .
$$


In the Cracow model, the final expression for $\Delta N$ (the number of particles in the rapidity interval $\Delta y)$ is

$$
\begin{aligned}
\Delta N & =\int d \Sigma_{\mu} n U^{\mu}=\Delta y \pi r_{\max }^{2} \tau_{f}\left(n_{\text {norm }}+\frac{N_{\text {cond }}}{\mathcal{V}}\right) \\
& \equiv \Delta y \frac{d V}{d y}\left(n_{\text {norm }}+\frac{N_{\text {cond }}}{\mathcal{V}}\right) .
\end{aligned}
$$

Consequently, for the rapidity density one gets

$$
\frac{\Delta N}{\Delta y}=\frac{d V}{d y} n_{\text {norm }}+\frac{d V}{\mathcal{V} d y} N_{\text {cond }}
$$

In our case $\mathcal{V}=\pi r_{\max }^{2} \tau_{f} \Delta y_{\text {cond }}$, where $\Delta y_{\text {cond }}$ is the rapidity range where the condensate may be formed. In this work we assume $\Delta y_{\text {cond }}=1$. Consequently we get

$$
\frac{\Delta N}{\Delta y}=\frac{d V}{d y} n_{\text {norm }}+N_{\text {cond }}
$$

\section{B. The particle spectra with the local condensate}

The momentum distribution is obtained from the formula

$$
E_{p} \frac{d N}{d^{3} p}=\frac{d N}{d y d \phi_{p} p_{T} d p_{T}}=\int d \Sigma_{\mu} p^{\mu} \bar{f}(x, p),
$$

where the condensate part of the distribution function can be written as

$$
\delta\left(p_{X}\right) \delta\left(p_{Y}\right) \delta\left(p_{Z}\right)=\frac{1}{p_{T} m^{2}} \delta\left(\sinh \theta_{T}-\frac{p_{T}}{m}\right) \delta\left(\phi-\phi_{p}\right) \delta\left(\eta_{\|}-y\right) .
$$

In our previous work we have found that the Cracow single freeze-out model can describe the LHC data for 2.76 TeV Pb+Pb collisions very well [31, 32]. Therefore, at first we test effects of the Bose condensation using this model. In this case $\sinh \left(\theta_{T}\right)=r / \tau_{f}$, where $r$ and $\tau_{f}$ are the freeze-out radius and proper time, respectively. Using this relation we may further write

$$
\delta\left(p_{X}\right) \delta\left(p_{Y}\right) \delta\left(p_{Z}\right)=\frac{1}{p_{T} m} \delta\left(p_{T}-r \frac{m}{\tau_{f}}\right) \delta\left(\phi-\phi_{p}\right) \delta\left(\eta_{\|}-y\right)
$$

Using this result we obtain the final expression for the momentum distribution

$$
\begin{aligned}
\frac{d N}{d y d \phi_{p} p_{T} d p_{T}} & =\frac{g}{(2 \pi)^{3}} \int_{0}^{2 \pi} d \phi \int_{-\infty}^{\infty} d \eta_{\|} \int_{0}^{\infty} \theta\left(r_{\max }-r\right) r d r \\
& \times\left[m_{T} \sqrt{\tau_{f}^{2}+r^{2}} \cosh \left(\eta_{\|}-y\right)-p_{T} r \cos \left(\phi-\phi_{p}\right)\right] \\
& \times\left\{\exp \left(\frac{\mu}{T}-\frac{1}{T}\left[m_{T} \sqrt{1+\frac{r^{2}}{\tau_{f}^{2}}} \cosh \left(\eta_{\|}-y\right)-p_{T} \frac{r}{\tau_{f}} \cos \left(\phi-\phi_{p}\right)\right]\right) \mp 1\right\}^{-1} \\
& \times\left[1+\frac{(2 \pi)^{3}}{\mathcal{V}} \frac{\tau_{f}}{p_{T} m^{2}} \delta\left(r-p_{T} \frac{\tau_{f}}{m}\right) \delta\left(\phi-\phi_{p}\right) \delta\left(\eta_{\|}-y\right)\right]
\end{aligned}
$$


where $\theta\left(r_{\max }-r\right)$ is the Heaviside step function.

The first term in the (31) is the usual one, while the second term with the delta function corresponds to the Bose condensate. The integrals over $\phi$ and $\eta_{\|}$are cancelled by the delta functions and we obtain:

$$
\begin{aligned}
\left.\frac{d N}{d y d \phi_{p} p_{T} d p_{T}}\right|_{\text {cond }}= & \frac{g}{\mathcal{V}} \int_{0}^{\infty} \theta\left(r_{\max }-r\right) r d r\left[m_{T} \sqrt{\tau_{f}^{2}+r^{2}}-p_{T} r\right] \frac{\tau_{f}}{p_{T} m^{2}} \delta\left(r-p_{T} \frac{\tau_{f}}{m}\right) \\
& \times\left\{\operatorname { e x p } \left(\frac{\mu}{T}-\frac{1}{T}\left[m_{T} \sqrt{\left.\left.\left.1+\frac{r^{2}}{\tau_{f}^{2}}-p_{T} \frac{r}{\tau_{f}}\right]\right) \mp 1\right\}^{-1}}\right.\right.\right. \\
& =\frac{g}{\mathcal{V}} \frac{\tau_{f}^{3}}{m^{2}} \theta\left(r_{\max }-p_{T} \tau_{f} / m\right)\left\{\exp \left(\frac{m-\mu}{T}\right) \mp 1\right\}^{-1} \\
& =\frac{1}{\mathcal{V}} \frac{\tau_{f}^{3}}{m^{2}} \theta\left(r_{\max }-p_{T} \tau_{f} / m\right) N_{\text {cond }},
\end{aligned}
$$

where $N_{\text {cond }}$ is the number of particles in Bose condensate as in (3). It follows from equation (32) that Bose condensate adds a constant number of particles to the usual momentum spectrum and these particles may have a momentum up to

$$
p_{T}^{\max }=m \frac{r_{\max }}{\tau_{f}}
$$

We added the zero momentum level into THERMINATOR using the Dirac delta function (30). It turns out that in order to reproduce the spectra we may keep the same $r_{\max } / \tau_{f}$ ratio as in our previous paper [32] and just rescale $r_{\max }$ and $\tau_{f}$ in such a way that we obtain the volume determined from the studies of multiplicities described in Sec. IV. The results for pions are shown in Fig. 5. The error bars for $\pi^{ \pm}$are not shown, because they are of the order of the symbol size.

One may notice a very good overall agreement. In the BEC model the spectra of other particles including protons, kaons, $K^{*}(892)^{0}$ and $\phi(1020)$, also agree with data, and are almost identical to those that we obtained in [32]. The agreement with data for pions at high $p_{T}$ is even improved compared to [32], because we use the same $r_{\max } / \tau_{f}$, but have bigger temperature in BEC than in NEQ. Therefore there are more high- $p_{T}$ particles in BEC than in NEQ.

On the other hand, the agreement of pions in the EQ model with the data is rather good, but other particles are described much worse [32]. For example, protons in EQ model deviate from data for more than the factor of two in the most central collisions at low $p_{T}[35]$.

The largest difference for pions is also seen at low $p_{T}$. There is a step at $p_{T}=p_{T}^{\max }$ in the BEC model, see Eq. (33), because of the contribution of the particles from the ground 

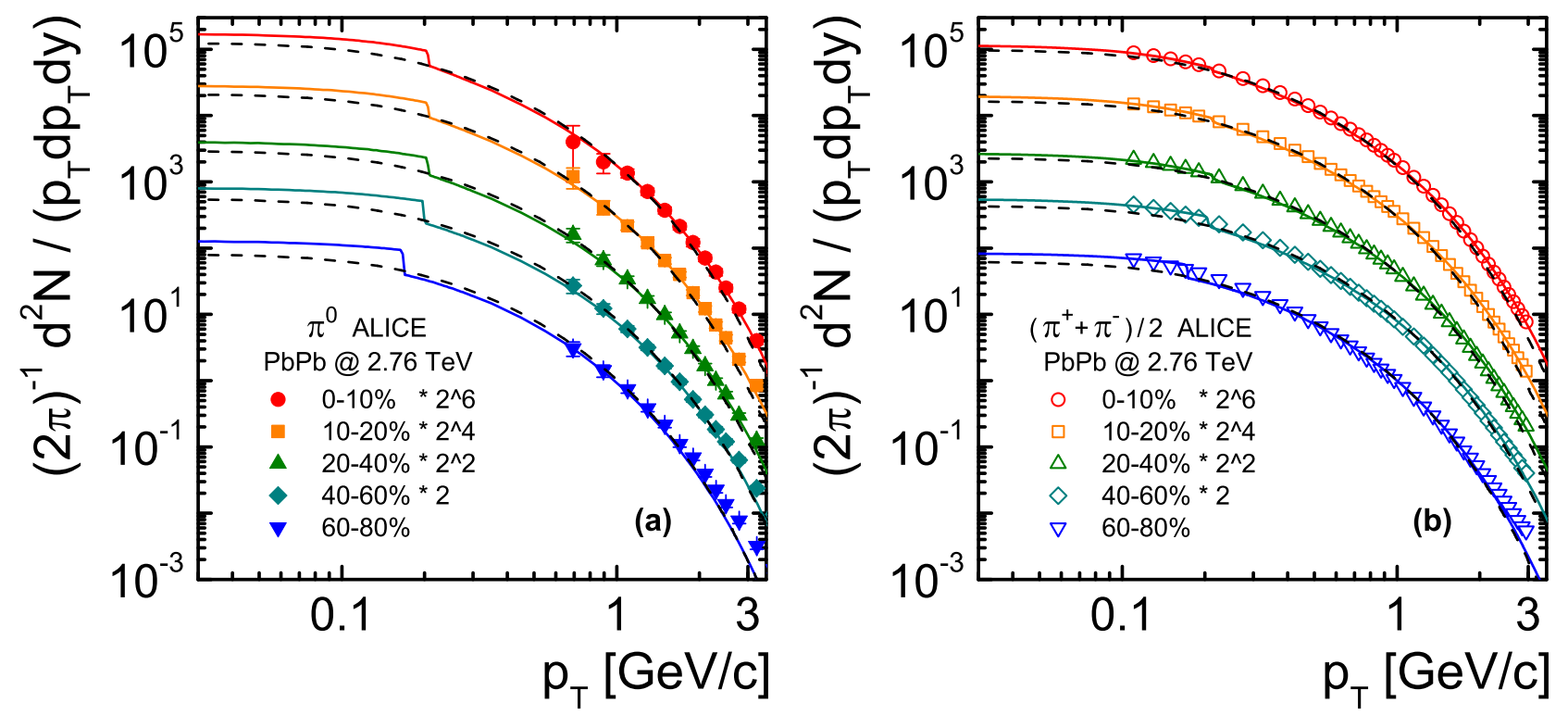

FIG. 5: (Color online) The $p_{T}$ spectra calculated in the BEC model (solid lines) and EQ model (dashed lines) at different centralities are compared with the existing data (symbols) for neutral pions [42] on the left and with the data for charged pions [26] on the right.

state. This is the artefact of the approximation that all particles from the low discrete levels are accumulated in the ground state (3). The step is larger for neutral pions, because they are lighter and therefore condense earlier. Unfortunately, $\pi^{0}$ 's have not been measured yet at low $p_{T}$. There are only several points for charged pions, $\pi^{ \pm}$, which are plotted in the linear scale without the $2^{n}$ factors in Fig. 6. One can see that the EQ lines do not describe the data in the low $p_{T}$ region for the very peripheral collisions. For the most central collisions the EQ lines go along lower edges of the error bars. This cannot be improved, because the EQ model overshoots protons at these centralities [32, 35]. Therefore it would be impossible to describe in EQ neither low $p_{T}$ pions nor protons when the experiment provides the improved data with lower error bars.

The BEC line with the step is within the error bars for the most central collisions, but the step is too big for more peripheral collisions. However, the smallest $p_{T}$ points are fitted at all centralities. One may also notice that the amount of extra particles to the left of the step is approximately equal to the number of missing particles to the right of the step. Therefore the inclusion of several more levels would lead to finer steps and smoother line, which goes within the error bars.

The grey bands indicate the maximal and minimal amount of condensate at the $10 \%$ higher 

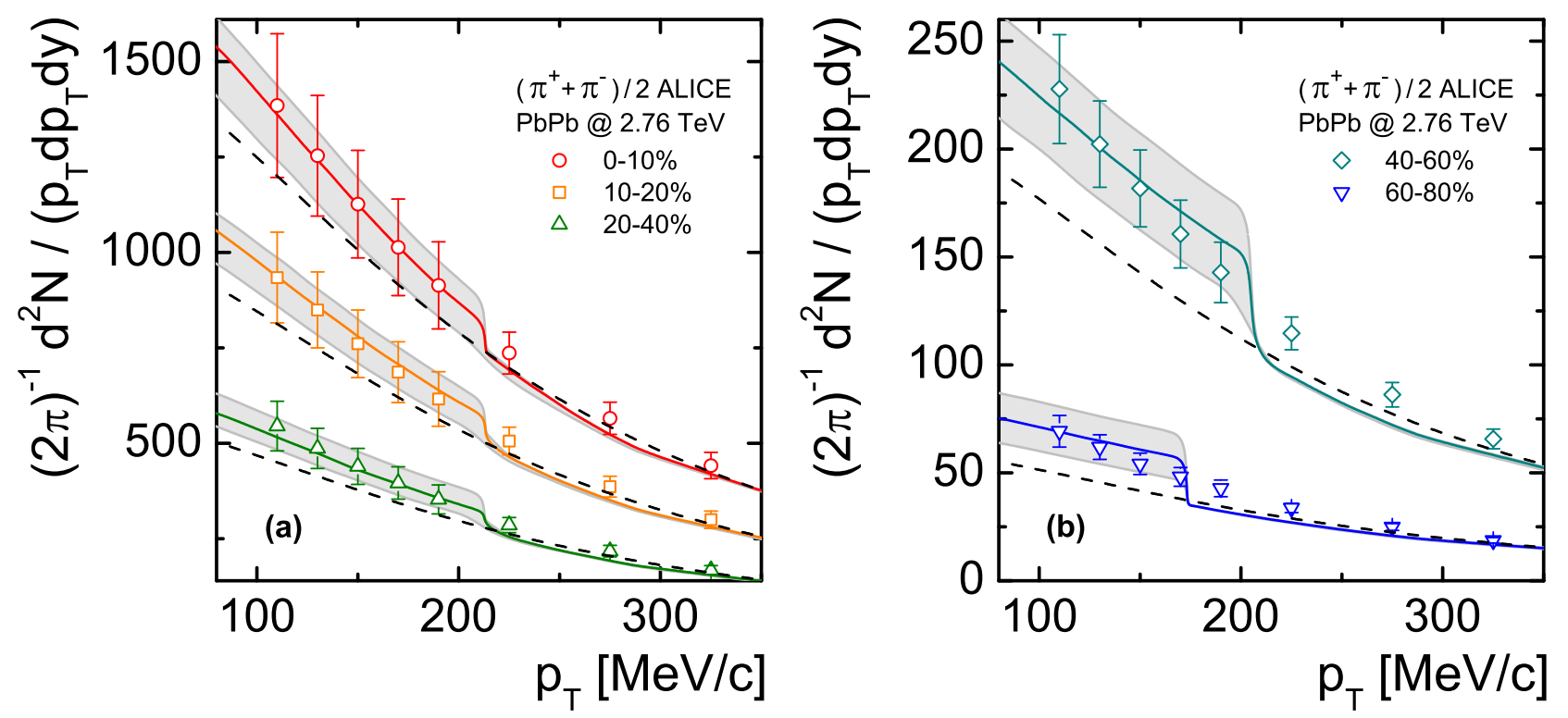

FIG. 6: (Color online) The low momentum part of the spectra for charged pions in central and semicentral collisions on the left and in peripheral collisions on the right are compared to the calculations in the BEC (solid lines) and EQ (dashed lines) models. The grey bands correspond to minimal and maximal condensate rate shown in Fig. 4, which is obtained assuming $10 \%$ increase of the $\chi^{2} / N_{\text {dof }}$, see Fig. 3 right.

$\chi^{2} / N_{\text {dof }}$ from the best fit, see Fig. 3 right and Fig. 4 . The grey band is within the error bars for most central collisions. One can even double the contribution from condensate there. However, in the most peripheral collisions the upper bound for the condensate is definitely too high and should be decreased by a factor of two ${ }^{3}$. Thus, the combined data on mean multiplicities and spectra are compatible even with the constant condensate rate at the level of $5 \%$.

\section{CONCLUSIONS}

In this work we have analysed the possibility of the pion condensation in heavy-ion collisions at the LHC energies. This phenomenon has attracted a lot of attention in the past (see [46] and references therein).

Our study shows that the pion condensate rate in the present data is $2 \%$ in the most central collisions and $7-8 \%$ in peripheral collisions (with a sudden increase to $19 \%$ in the most

${ }^{3}$ One should remember that the discussed low- $p_{T}$ region of $\pi^{ \pm}$spectra gives a small contribution to the mean multiplicities, therefore the spectra are much more sensitive to the condensate. 
peripheral collisions). The obtained $\chi^{2} / N_{\text {dof }}$ values for the thermodynamic parameters have a shallow minimum. It results in a large uncertainty in the determination of the exact number of pions in the condensate. The spectra show an opposite trend with centrality allowing larger condensate contributions in the most central collisions as compared to the peripheral ones.

The data for charged pion spectrum at the 80-90\% centrality would help to confirm the increase of the condensate rate with centrality. However, the most important are the measurement of the $\pi^{0}$ multiplicities and the $\pi^{0}$ spectra in the low $p_{T}$ region.

\section{Acknowledgments}

We thank Mateusz Ploskon for sending us the data on $\pi^{0}$ spectra. V.B and W.F. were supported by Polish National Science Center grant No. DEC-2012/06/A/ST2/00390.

[1] P. Koch, J. Rafelski, South Afr. J. Phys. 9, 8 (1986).

[2] J. Cleymans and H. Satz, Z. Phys. C 57, 135 (1993).

[3] J. Sollfrank, M. Gazdzicki, U. W. Heinz, J. Rafelski, Z. Phys. C 61, 659 (1994).

[4] E. Schnedermann, J. Sollfrank, U. W. Heinz, Phys. Rev. C 48, 2462 (1993).

[5] P. Braun-Munzinger, J. Stachel, J. P. Wessels, N. Xu, Phys. Lett. B 344, 43 (1995).

[6] F. Becattini, Z. Phys. C 69, 485 (1996).

[7] P. Braun-Munzinger, J. Stachel, J. P. Wessels, N. Xu, Phys. Lett. B 365, 1 (1996).

[8] J. Cleymans, D. Elliott, H. Satz, R. L. Thews, Z. Phys. C 74, 319 (1997).

[9] F. Becattini and U. W. Heinz, Z. Phys. C 76, 269 (1997) [Erratum-ibid. C 76, 578 (1997)].

[10] F. Becattini, J. Phys. G 23, 1933 (1997).

[11] G. D. Yen, M. I. Gorenstein, Phys. Rev. C 59, 2788 (1999).

[12] J. Cleymans, K. Redlich, Phys. Rev. Lett. 81, 5284 (1998).

[13] M. Gazdzicki, M. I. Gorenstein, Acta Phys. Polon. B 30, 2705 (1999).

[14] J. Letessier and J. Rafelski, Phys. Rev. C 59, 947 (1999).

[15] P. Braun-Munzinger, I. Heppe, J. Stachel, Phys. Lett. B 465, 15 (1999).

[16] J. Cleymans and K. Redlich, Phys. Rev. C 60, 054908 (1999).

[17] F. Becattini, J. Cleymans, A. Keranen, E. Suhonen, K. Redlich, Phys. Rev. C 64, 024901 (2001).

[18] P. Braun-Munzinger, D. Magestro, K. Redlich, J. Stachel, Phys. Lett. B 518, 41 (2001). 
[19] W. Florkowski, W. Broniowski, M. Michalec, Acta Phys. Polon. B 33, 761 (2002).

[20] W. Broniowski and W. Florkowski, Phys. Rev. Lett. 87, 272302 (2001).

[21] W. Broniowski, W. Florkowski, Phys. Rev. C 65, 064905 (2002).

[22] W. Broniowski, A. Baran and W. Florkowski, AIP Conf. Proc. 660, 185 (2003).

[23] F. Retiere and M. A. Lisa, Phys. Rev. C 70, 044907 (2004).

[24] P. Braun-Munzinger, K. Redlich and J. Stachel, In R. C. Hwa, X. N. Wang Quark gluon plasma World Scientific Publishing, 2004, 491-599 [nucl-th/0304013].

[25] F. Becattini, M. Gazdzicki, A. Keranen, J. Manninen, R. Stock, Phys. Rev. C 69, 024905 (2004).

[26] B. Abelev et al. [ALICE Collaboration], Phys. Rev. C 88, 044910 (2013).

[27] J. Stachel, A. Andronic, P. Braun-Munzinger and K. Redlich, J. Phys. Conf. Ser. 509, 012019 (2014).

[28] C. Gale, S. Jeon, B. Schenke, P. Tribedy and R. Venugopalan, Phys. Rev. Lett. 110, 012302 (2013).

[29] B. Abelev et al. [ALICE Collaboration], Phys. Rev. Lett. 109, 252301 (2012).

[30] E. Molnar, H. Holopainen, P. Huovinen and H. Niemi, Phys. Rev. C 90, no. 4, 044904 (2014).

[31] V. Begun, W. Florkowski and M. Rybczynski, Phys. Rev. C 90, no. 1, 014906 (2014).

[32] V. Begun, W. Florkowski and M. Rybczynski, Phys. Rev. C 90, no. 5, 054912 (2014).

[33] I. Melo and B. Tomasik, arXiv:1502.01247 [nucl-th].

[34] B. B. Abelev et al. [ALICE Collaboration], Phys. Rev. C 89, no. 2, 024911 (2014).

[35] V. Begun, arXiv:1412.6532 [nucl-th].

[36] M. Petran, J. Letessier, J. Rafelski and G. Torrieri, Comput. Phys. Commun. 185, 2056 (2014).

[37] A. Kisiel, T. Taluc, W. Broniowski and W. Florkowski, Comput. Phys. Commun. 174, 669 (2006).

[38] M. Chojnacki, A. Kisiel, W. Florkowski and W. Broniowski, Comput. Phys. Commun. 183, 746 (2012).

[39] B. B. Abelev et al. [ALICE Collaboration], Phys. Rev. C 91, no. 2, 024609 (2015).

[40] B. B. Abelev et al. [ALICE Collaboration], Phys. Rev. Lett. 111, 222301 (2013).

[41] B. B. Abelev et al. [ALICE Collaboration], Phys. Lett. B 728, 216 (2014).

[42] B. B. Abelev et al. [ALICE Collaboration], Eur. Phys. J. C 74, no. 10, 3108 (2014).

[43] V. V. Begun and M. I. Gorenstein, Phys. Rev. C 77, 064903 (2008).

[44] F. Becattini, M. Bleicher, E. Grossi, J. Steinheimer and R. Stock, Phys. Rev. C 90, no. 5, 054907 (2014). 
[45] W. Florkowski, "Phenomenology of Ultra-Relativistic Heavy-Ion Collisions," (World Scientific, Singapore, 2010).

[46] E. Shuryak, arXiv:1412.8393 [hep-ph]. 